\title{
A Full-Newton Step Interior Point Method for Fractional Programming Problem Involving Second Order Cone Constraint
}

\author{
Ali Sadeghi ${ }^{1}$, Mansour Saraj ${ }^{2 *}$, \\ Nezam Mahdavi Amiri ${ }^{3}$
}

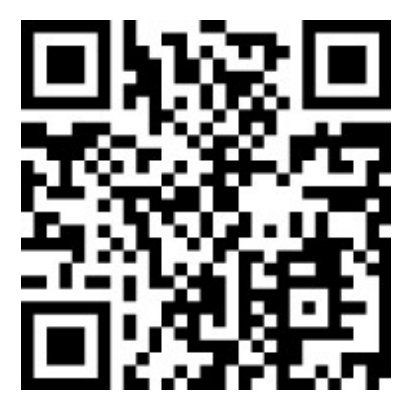

${ }^{*}$ Corresponding author

1. Department of Mathematics, Faculty of Mathematical Sciences and Computer, Shahid Chamran University of Ahvaz,Ahvaz-IRAN, a-sadeghi@phdstu.scu.ac.ir

2. Department of Mathematics, Faculty of Mathematical Sciences and Computer,

Shahid Chamran University of Ahvaz, Ahvaz-IRAN, msaraj@scu.ac.ir

3. Faculty of Mathematical Sciences, Sharif University of Technology, Tehran, Iran, nezamm@sharif.edu

\begin{abstract}
Some efficient interior-point methods (IPMs) are based on using a self-concordant barrier function related to the feasibility set of the underlying problem. Here, we use IPMs for solving fractional programming problems involving second order cone constraints. We propose a logarithmic barrier function to show the self concordant property and present an algorithm to compute $\varepsilon$-solution of a fractional programming problem. Finally, we provide a numerical example to illustrate the approach.
\end{abstract}

Key Words: Fractional programming; Self concordant; Logarithm barrier function.

Mathematical Subject Classification: 90C32, 90C51.

\section{Introduction}

Since the seminal work of Karmarkar (1984) in 1984, interior-point methods (IPMs) have shown to be very useful in many areas of optimization. Among various approaches for solving optimization and mathematical problems, IPMs have shown to be competitive due to polynomial complexity and computational performance. Using the theory of self-concordant (SC) functions and SC barrier functions (SCBFs), IPMs were introduced by Nesterov and Nemirovski (1989). In the early 90's of the past century, this theory was a breakthrough and provided a unified framework for the design and analysis of IPMs for a large class of convex optimization problems, yielding thebest known polynomial complexity results Schmieta(2003).

Surveys on the theory and practice of IPMs can be found in the recent books related to the subject by Boyd and Vandenberghe (1998) and Roos etal. (2005a). Roos (2006) investigated an $O(n)$ full Newton step for linear optimization (LO). His algorithm constructs strictly feasible iterates for a sequence of perturbations of the given problem. A significant feature of this algorithm is its use of only full-Newton steps, namely full Newton steps being composed of a feasibility step and a centering step. A full Nesterov-Todd step of Zangiabadi(2013) has lead to a primal-dual interior point algorithm for second order cone optimization. It has been shown that the algorithm either finds a solution in a finite number of iterations or determines that the primal-dual problem pair has no optimal solution with vanishing duality gap. Alizadeh (2003) showed that Euclidean Jordan algebras underly the analysis of IPMs for optimization 
over symmetric cones. Several IPMs are based on following the so-called central path of the underlying problem as a guideline to obtain an $\varepsilon$-solution of the problem. The main source in such methods is usually some variant of the Newton method. Another key element in the design and analysis of IPMs is a barrier function for the domain of the problem. Until recently, most IPMs were based on the use of the classical logarithmic barrier function. The best known iteration bound for LO is attained by a full-Newton step IPM (FNS-IPM) based on the logarithmic barrier function, mathematically given by

$$
O\left(\sqrt{n} \ln \frac{n}{\varepsilon}\right)
$$

where $n$ denotes the number of inequalities in the problem and $\varepsilon$ is the desired accuracy. Feng et al. (2014) proposed a predictor-corrector path-following interior-point algorithm for semi-definite optimization problems. Their algorithm contains the low iteration bound of $O(\sqrt{n} L)$ as compared to $O(n L)$ due to the usual wide neighborhood algorithm. The seminal work of Nesterov and Nemirovski (1989) made it clear that the reason for the prominent role of the logarithmic barrier function in $\mathrm{LO}$ and convex optimization is the function being self-concordant.

In the past few decades, fractional programming problems have attracted the interests of many researchers. These problems have applications in many real physical problems such as finance, production planning, electronic, etc. Fractional programs are being used for modelling real life problems involving one or more objective(s) such as actual cost/standard cost, inventory/sales and profit/cost. There are different algorithms to determine solutions of particular fractional programming problems. For example, Charnes and Cooper (1962) converted a linear fractional program (LFP) to a linear program by a variable transformation technique. Tantawy (2008) proposed an iterative method based on a conjugate gradient projection approach.

Our main goal here is to present and make use of a logarithmic function related to fractional optimization problems. We prove that the logarithmic barrier function is SC and therefore an IPM can be used to solve and obtain an $\varepsilon-$ solution of the problem. The remainder of our work is organized as follows. In Section 2, we recall some well-known results of self concordant barrier functions (SCBF). We also deal with the problem of minimizing a linear fractional function over a closed convex set with nonempty (relative) interior. In Section 3, the formal definition of an SCBF is given and we outline the related FNS-IPM. Then, we establish an iteration bound of the algorithm. In Section 4, an example practical is worked throgh to illustrate our approach.

We note that we have restricted ourselves to fractional programming problem with second order cone constraint, although the extension to the more general class of symmetric optimization problems is nowadays more or less straightforward.

\section{Preliminaries}

We first recall the notion of a self-concordant barrier function (SCBF) $\varphi: D \rightarrow R$, where $D$ is an open convex subset of $R^{n}$. We start by considering the case with $n=1$. Then, $\varphi$ is a univariate function and its domain $D$ is an open interval in $R$. One calls $\varphi$ as a $\kappa$-SCBF, if there exists a nonnegative number $\kappa$ such that

$$
\left|\varphi^{\prime \prime \prime}(x)\right| \leq 2 \kappa\left(\varphi^{\prime \prime}(x)\right)^{\frac{3}{2}}, \quad \forall x \in D .
$$

Note that this definition assumes that $\varphi$ is three times differentiable. Moreover, it implies that $\varphi^{\prime \prime}(x)$ is nonnegative, and hence $\varphi$ is convex. The most well-known example of an SCBF is $-\ln t$, Which is a $\kappa$-SCBF on the positive real axis.

Now, suppose that $\varphi$ is a multivariate function, i.e., $n>1$. Then, $\varphi$ is called $\kappa$-SCBF, if its restriction to an arbitrary line intersecting $D$ is a $\kappa$-SCBF. In other words, $\varphi$ is a $\kappa$-SCBF, if (2) holds when we replace all derivatives in (2) by directional derivatives, for every direction $h \in R^{n}$. More precisely, denoting these directional derivatives by $\nabla^{2} \varphi(x)[h, h]$ and $\nabla^{3} \varphi(x)[h, h, h]$, respectively, $\varphi$ is $\kappa$-SCBF if and only if

$$
\left|\nabla^{3} \varphi(x)[h, h, h]\right| \leq 2 \kappa\left(\nabla^{2} \varphi(x)[h, h]\right)^{\frac{3}{2}}, h \in R^{n}, \forall x \in D .
$$

The $\kappa$-self-concordancy condition bounds the third order term in terms of the second order term in the Taylor expansion. Hence, if it is satisfied, it means that the second order Taylor expansion locally provides a good quadratic approximation of $\varphi(x)$. This latter property ensures that the Newton method behaves well on self-concordant functions, as being shown later on. Recall that the definition of the $\kappa$-self-concordance property applies to every three 
times differentiable convex function with an open domain.

Proposition 2.1. (Nesterov and Nemirovskii (1989))

(a) (addition and scaling) Let $\varphi_{i}$ be $\kappa_{i}$-self-concordant on $\digamma_{i}^{0}, i=1,2$, and $\rho_{1}, \rho_{2} \in R^{+}$. Then, $\rho_{1} \varphi_{1}+\rho_{2} \varphi_{2}$ is $\kappa$-self-concordant on $\digamma_{1}^{0} \cap \digamma_{2}^{0}$, where $\kappa=\max \left\{\frac{\kappa_{1}}{\sqrt{\rho_{1}}}, \frac{\kappa_{2}}{\sqrt{\rho_{2}}}\right\}$.

(b) (affine invariance) Let $\varphi$ be $\kappa$-self-concordant on $\digamma^{0}$ and let $\beta(y)=B y+b: R^{k} \longrightarrow R^{m}$ be an affine mapping such that $B\left(R^{k}\right) \cap \digamma^{0} \neq \emptyset$. Then, $\varphi(B()$.$) is \kappa$-self-concordant on $\left\{y: ß(y) \in \digamma^{0}\right\}$.

Corollary 2.1. Let

$$
G=\left\{x \in R^{n} \mid A x-b \leq 0\right\}=\left\{x \in R^{n} \mid a_{i}^{T} x-b_{i} \leq 0, i=1, \ldots, m\right\}
$$

be a convex polyhedron defined by a set of linear inequalities satisfying the Slater condition:

$$
\exists x \in R^{n}: a_{i}^{T} x-b_{i}<0, i=1, \ldots, m
$$

Then, the standard logarithmic barrier for $G$, given by

$$
F(x)=-\sum_{i=1}^{m} \ln \left(b_{i}-a_{i}^{T} x\right)
$$

is self-concordant on the interior of $G$.

Proof. From the Slater condition it follows that

$$
G=\left\{x \in R^{n} \mid a_{i}^{T} x-b_{i}<0, i=1, \ldots, m\right\}=\bigcap_{i=1}^{m} g_{i}, \quad g_{i}=\left\{x \in R^{n} \mid a_{i}^{T} x-b_{i}<0\right\}
$$

Since the function $-\ln t$ is self-concordant on the positive half-axis, every function $F_{i}(x)=-\ln \left(b_{i}-a_{i}^{T} x\right)$ is selfconcordant on $g_{i}$ (item (a) of proposition 2.1); note that $g_{i}$ is the inverse image of the positive half-axis under the affine mapping $x \longmapsto b_{i}-a_{i}^{T} x$, when $F(x)=\sum_{i=1}^{m} F_{i}(x)$ is self-concordant on $G=\bigcap_{i=1}^{m} g_{i}$ (item (b) of proposition 2.1). Due to proposition 2.1, it is clear that if $\varphi$ is $\kappa$-self-concordant with $\kappa>0$, then $\varphi$ can be re-scaled by a positive scalar so that it becomes 1-self-concordant, meaning that for a positive constant $\lambda, \lambda \varphi$ is $\frac{\kappa}{\sqrt{\lambda}}$-self-concordant.

\subsection{Minimizing Linear Fractional Problem on Second Order Cone Constraint}

A typical second-order cone in $R^{n}$ has the form

$$
Q=\left\{x=\left(x_{0} ; x_{1} ; \ldots ; x_{n-1}\right) \in R^{n}: x_{0}^{2} \geq \sum_{i=1}^{n-1} x_{i}^{2}\right\}
$$

or

$$
\left\{x=\left(x_{0} ; \bar{x}\right) \in R^{n}: x_{0} \geq\|\bar{x}\|\right\}
$$

where $n \geq 1$ is a natural number.

Consider the following problem

$$
\begin{aligned}
(P 1): \quad \operatorname{Max} & F(x)=\frac{c^{T} x+\alpha}{d^{T} x+\beta} \\
& A x \leq b, \\
& x \in Q
\end{aligned}
$$

where $x \in R^{n}, c=\left(c_{1}, c_{2}, \ldots, c_{n}\right)^{T} \in R^{n}$, and $\alpha$ and $\beta$ are real scalars, $A$ is an $m \times n$ matrix, $c, d \in R^{n}$, and $b \in R^{m}$. It is assumed that the feasible set is a bounded and closed set and thus is compact. Moreover, $d^{T} x+\beta>0$, 
for all $x$ in the feasible region.

We can reformulate problem $(P 1)$ as follows:

$$
\begin{gathered}
(P 2): \text { Min } x_{n+1} \\
A x \leq b, \\
c^{T} x+\alpha-x_{n+1}\left(d^{T} x+\beta\right) \leq 0, \\
x \in Q .
\end{gathered}
$$

We can then define the logarithmic barrier function for $(P 2)$ to be

$$
\Phi(x, \mu)=\frac{x_{n+1}}{\mu}-\sum_{j=1}^{m} \ln \left(b_{i}-a_{i}^{T} x\right)-\ln (\operatorname{det}(x))-\ln \left(-\left(c^{T} x+\alpha\right)+x_{n+1}\left(d^{T} x+\beta\right)\right),
$$

where $\operatorname{det}(x)=x_{0}^{2}-\|\bar{x}\|^{2}$. The following results are well-known.

Lemma 2.1. A symetric matrix $X \in R^{n \times n}$ is positive semi-definite if and only if there exists a matrix $C$ such that $X=C C^{T}$.

Theorem 2.2. The logarithmic barrier function $q(x)=-\ln \left(x^{T} A^{T} A x+b^{T} x+\beta\right)$ is self-concordant on $\left\{x \mid x^{T} A^{T} A x+\right.$ $\left.b^{T} x+\beta>0\right\}$. Also, $-\ln \left(x^{T} H x+b^{T} x+\beta\right)$ is self-concordant on $x^{T} H x+b^{T} x+\beta>0$, when $H$ is positive semidefinite.

Proof. Note that $-\ln (A x+B)$ (item (a) of proposition 2.1) and $-2 \ln (A x+B)$ are self-concordant (item (b) of proposition 2.1). Then, $-\ln \left((A x+B)^{T}(A x+B)\right)$ is $\frac{1}{\sqrt{2}}$-self-concordant. Also, $q(x)$ is self-concordant. Then, we conclude by Lemma 2.1 that $-\ln \left(x^{T} H x+b^{T} x+c\right)$ is self-concordant when matrix $H$ is positive semi-definite.

Corollary 2.2. The logarithmic barrier function $\ln (g(x))=\ln \left[-\left(c^{T} x+\alpha\right)+x_{n+1}\left(d^{T} x+\beta\right)\right]$ is self-concordant on $\left(c^{T} x+\alpha\right)-x_{n+1}\left(d^{T} x+\beta\right)<0$.

Proof. $\ln (g(x))$ is self-concordant because $g(x)$ is a convex quadratic function, that is,

$$
g(x)=x^{T} H x+b^{T} x+c,
$$

with

$$
H=\left(\begin{array}{ccccc}
0 & 0 & \ldots & 0 & d_{1} \\
0 & 0 & \ldots & 0 & d_{2} \\
\vdots & \vdots & \ddots & \vdots & \vdots \\
0 & 0 & \ldots & 0 & d_{n} \\
d_{1} & d_{2} & \ldots & d_{n} & 0
\end{array}\right)
$$

and

$$
b^{T}=\left[-\left(c_{1}-x_{n+1} d_{1}\right),-\left(c_{2}-x_{n+1} d_{2}\right), \ldots,-\left(c_{n}-x_{n+1} d_{n}\right), d^{T} x+\beta\right],
$$

where $H$ is an $(n+1) \times(n+1)$ positive semidefenite matrix and $b^{T}$ is a $1 \times(n+1)$ vector. Then, by Theorem 2.2 and Corollary 2.1 we have that $\ln (g(x))$ is self-concordant.

Lemma 2.2. Let $g: R \rightarrow R$ be a convex function defined on $R_{+}$and

$$
\left|g^{\prime \prime \prime}(x)\right| \leq 3 \frac{g^{\prime \prime}(x)}{x} .
$$

Then, $f(x)=-\ln (-g(x))-\ln x$ is self-concordant on $\{x \mid x>0, g(x)<0\}$.

Theorem 2.3. The logarithmic barrier function $\Phi_{1}$ is self-concordant. 
Proof. We must first show that the function $-\ln (\operatorname{det}(x))$ is self-concordant on $\{(y, x) \mid\|x\|<y\}$. We write $f$ as $f(y, x)=-\ln \left(y-\frac{x^{T} x}{y}\right)$ and restrict the function to a line $x=\hat{x}+t v, y=\hat{y}+t w$,

$$
f(\hat{y}+t w, \hat{x}+t v)=-\ln \left(\hat{y}+t w-\frac{\hat{x}^{T} \hat{x}}{\hat{y}+t w}-\frac{2 t \hat{x}^{T} v}{\hat{y}+t w}-\frac{t^{2} v^{T} v}{\hat{y}+t w}\right) .
$$

If $w=0$, then it is obvious that the argument of the log function reduces to a quadratic function in $t$, posing a simple case. Otherwise, we can use $y$ instead of $t$ by a change of variable $t=\frac{y-\hat{y}}{w}$. We therefore obtain

$$
f(\hat{y}+t w, \hat{x}+t v)=-\ln \left(\alpha+\beta y-\frac{\gamma}{y}\right)-\ln (y),
$$

where

$$
\alpha=2 \frac{y v^{T} v}{w^{2}}-2 \frac{\hat{x}^{T} v}{w}, \beta=1-\frac{v^{T} v}{w}, \gamma=\hat{x}^{T} \hat{x}-2 \frac{y \hat{x}^{T} v}{w}-2 \frac{y^{2} v^{T} v}{w^{2}} .
$$

Defining $g(y)=-\alpha-\beta y+\frac{\gamma}{y}$, we have

$$
f(\hat{y}+t w, \hat{x}+t v)=-\ln (-g(y))-\ln (y) .
$$

The function $\mathrm{g}$ is convex since $\gamma>0$ and $g$ satisfies (3) because

$$
g^{\prime \prime \prime}=\frac{-6 \gamma}{y^{4}}, \quad g^{\prime \prime}(x)=\frac{2 \gamma}{y^{3}} .
$$

By using Corollary 2.1, Corollary 2.2 and Theorem 2.2, the proof is complete.

\section{Properties of Newton's Method}

Here, we assume that $\Phi_{1}(x, \mu)$, for some fixed $\mu>0$, is $\kappa$-self-concordant on its domain $\digamma$ with $\kappa>0$. Now, it can be concluded that if $\delta(x, \mu)$ is small enough, then the Newton process is quadratically convergent, where $\delta(x, \mu)=\sqrt{\Delta x H(x, \mu) \Delta x}$ and $H=\nabla^{2} \Phi_{B}(x, \mu)$.

Lemma 3.1. If $x$ is strictly primal feasible and $\mu>0$ such that $\delta=\delta(x, \mu) \leq \frac{1}{\kappa}$, then $x+\Delta x$ (where $\Delta x$ denotes the Newton step at $x$ ) is strictly feasible and

$$
\delta(x, \mu) \leq \frac{\kappa \delta^{2}}{(1-\kappa \delta)^{2}}
$$

Proof. see Klerk et al (2006).

\subsection{Logarithmic barrier algorithm with full Newton's steps}

We are now ready to state the algorithm using full Newton's steps.

Algorithm 1: Logarithmic barrier method using full Newton's steps

Input:

A proximity parameter $\tau, \quad 0 \leq \tau<1$;

an accuracy parameter $\varepsilon>0$;

$x^{0} \in \digamma^{0}$ and $\mu^{0}>0$ such that $\delta\left(x^{0}, \mu^{0}\right) \leq \tau ;$

a fixed barrier update parameter $\theta, 0<\theta<1$.

begin

$x:=x^{0} ; \mu:=\mu^{0} ;$

while $m \mu \geq 0$ do

begin

$\mu:=(1-\theta) \mu ; x:=x+\Delta x(\Delta x$ is the Newton step at $x)$

end

end 
Next, with the following result, we have the complexity of the algorithm.

Theorem 3.1. Let $x^{+}=x+\Delta x$ and $\mu^{+}:=(1-\theta) \mu$, where $\theta=\frac{1}{30 \kappa \sqrt{m}}$. Then,

$$
\delta(x, \mu) \leq \frac{1}{3 \kappa} \Rightarrow \delta\left(x^{+}, \mu^{+}\right) \leq \frac{1}{3 \kappa}
$$

Proof. See Roos et al. (2005a).

Theorem 3.2. If $\tau=\frac{1}{3 \kappa}$ and $\theta=\frac{1}{30 \kappa \sqrt{m}}$, then Algorithm 1 with full Newton's steps requires at most

$$
\left\lceil 30 \kappa \sqrt{m} \ln \frac{m \mu^{0}}{\varepsilon}\right\rceil
$$

iterations. The output is a strictly primal feasible and an $\varepsilon$-optimal solution.

Proof. By Theorem 3.1, the property $\delta(x, \mu) \leq \frac{1}{3 \kappa}$ is maintained in the course of the algorithm. Thus, each (full) Newton's step will yield a strictly feasible point, by Theorem 2.3 At each iteration the barrier parameter is reduced by the factor $1-\theta$. Hence, after $k$ iterations, we will have

$$
m \mu=(1-\theta)^{\kappa} m \mu^{0}
$$

Using this, one easily deduces that after no more than

$$
\left\lceil\frac{1}{\theta} \ln \frac{m \mu^{0}}{\varepsilon}\right\rceil
$$

iterations the algorithm will stop. Substitution of the value of $\theta$ in the theorem yields the desired bound.

\section{Numerical Example}

Generally, we know that linear fractional programming problems and fractional programming problems involving second order cone constraints are self-concordant functions. Here, we first define a logarithmic barrier function and then show that this function is $\frac{1}{\sqrt{2}}$-self-concordant, to which a full Newton's step interior point method can be applied. Consider the following linear fractional programming problem:

$$
\begin{array}{r}
(P 3): \quad \text { Max } z=\frac{2 x_{1}+x_{2}}{x_{1}+x_{2}+1} \\
3 x_{1}+x_{2} \leq 6, \\
x_{1}-x_{2} \leq 7, \\
x_{1}, x_{2} \geq 0 .
\end{array}
$$

The logarithmic barrier function for $(P 3)$ is given by

$$
\frac{x_{3}}{\mu}-\ln \left(6-3 x_{1}-x_{2}\right)-\ln \left(7-x_{1}+x_{2}\right)-\ln \left(x_{3}\left(x_{1}+x_{2}+1\right)-2 x_{1}-x_{2}\right)-\sum_{j=1}^{3} \ln \left(x_{j}\right) .
$$

Furthermore,

$$
\tau=\frac{1}{3 k}=\frac{\sqrt{2}}{3}, \theta=\frac{1}{30 k \sqrt{m}}=\frac{1}{30} .
$$

We choose $\varepsilon=0.5, \mu^{0}=0.8$ and $x^{0}=(1,1)$.

Then, the algorithm requires at most

$$
\left\lceil 30 \ln \frac{\mu^{0}}{\varepsilon}\right\rceil=14
$$

iterations. 


\section{Conclusion}

We showed that the logarithmic barrier function for fractional programming problem involving second order cone constraint is self concordant. Then, we presented an interior point method using full Newton's steps to solve such problems. We also established the computational complexity of the algorithm.

\section{Acknowledgment}

The authors are thankful to the Shahid Chamran University of Ahvaz and Sharif University of Technology for making this joint work possible and Shahid Chamran University of Ahvaz for the financial support.

\section{References}

1. Alizadeh, F. and Goldfarb, D. (2003). Second-order cone programming, Math. Program. Ser. B 95, 35-51.

2. Charnes, A. and Cooper, W. W. (1962). Programs with linear fractional functions, Naval Research Logistics Quarterly 9, 181-196.

3. Feng, Z. Z. and Fang, $L$.(2014). A new $O(\sqrt{n} L)$-iteration predictor-corrector algorithm with wide neighborhood for semi definite programming. J. Comput. Appl. Math. 256, 65-76.

4. Feng, Z. Z., Fang, L. and Guoping, H. (2014). An $O(\sqrt{n} L)$ iteration primal-dual path-following method, based on wide neighbourhood and large update, for second-order cone programming, Optimization 63(5), 679-691.

5. Karmarkar, N. K. (1984). A new polynomial-time algorithm for linear programming, Combinatorica 4, 373-395.

6. Kim, S. and Kojima, M. (2003). Exact solutions of some nonconvex quadratic optimization problems via SDP and SOCP relaxations, Computational Optimization and Applications 26, 143-154.

7. Klerk, E. de, Roos, C. and Terlaky, T. (2006). Nonlinear Optimization, University of Waterloo.

8. Lobo, M. S., Vandenberghe, L., Boyd L. and Lebret, H. (1998). Applications of second order cone programming, Linear Algebra Appl. 284, 193-228.

9. Nesterov, Y. E., and Nemirovsky, A. S. (1989). Self Concordant Functions and Polynomial Time Methods in Convex Programming, Report, Central Economical and Mathe-matical Institute, USSR Academy of Science, Moscow, USSR.

10. Renegar, J. (2001). A Mathematical View of Interior-Point Methods in Convex Optimization, MPS/SIAM Series on Optimization, Society for Industrial and Applied Mathematics (SIAM), Philadelphia, PA.

11. Roos, C. (2006). A full-Newton step $O(n)$ infeasible interior-point algorithm for linear optimization, SIAM J. Optim. 16(4), 1110-1136.

12. Roos, C., Terlaky, T. and Vial, J. P. (2005a). Theory and Algorithms for Linear Optimization, Springer, Chichester, UK, Theory and Algorithms for Linear Optimization, An Interior-Point Approach, John Wiley and Sons.

13. Schmieta, S. H. and Alizadeh, F. (2003). Extension of polynimal time interior-point algorithms to symmetric cones, Math. Program. 96(3), 409-438.

14. Tantawy, S. F. (2008). A new procedure for solving linear fractional programming problems, Journal of Mathematical and Computer Modeling 48, 969-973.

15. Zangiabadi, M., Gu, G. and Roos, C. (2013). A full nesterovtodd step infeasible interior-point method for second-order cone optimization, J. Optim. Theory Appl. 158, 816-858. 